\title{
Same-Day Sinus and Brain CT Imaging in the Medicare Population: Are Practice Patterns Changing in Association with Medicare Policy Initiatives?
}

\author{
(D) H. Kroll, (D) Ruszak Jr, (D). Hemingway, (DD. Hughes, and (D). Wintermark
}

\begin{abstract}
BACKGROUND AND PURPOSE: Monitoring the frequency of same-day sinus and brain CT (Outpatient Measure 14, "OP-14") is part of a recent large Centers for Medicare and Medicaid Services hospital outpatient quality initiative to improve imaging efficiency. This study investigates patient-level claims data in the Medicare population focusing on where same-day sinus and brain CT imaging is performed and how the frequency of same-day studies changed with time before and during OP-14 measure program implementation.
\end{abstract}

MATERIALS AND METHODS: Research Identifiable Files were used to identify all sinus and brain CT examinations from 2004 through 2012 for a $5 \%$ random patient sample of Medicare fee-for-service beneficiaries. Overall and site of service use rates were calculated for sameand non-same-day examinations. Changes were mapped to policy initiative timetables.

RESULTS: The number of same-day sinus and brain CT studies from 2004 to 2012 increased $67 \%$ from 1.85 (95\% Cl, 1.78-1.91) per 1000 Medicare beneficiaries in 2004 to 3.08 (95\% Cl, 3.00-3.15) in 2012. The biggest driver of increased same-day studies was the emergency department setting, from $0.56(95 \% \mathrm{Cl}, 0.53-0.60)$ per 1000 to $1.78(95 \% \mathrm{Cl}, 1.72-1.84 ;+215.7 \%)$. Overall use of brain $\mathrm{CT}$ from 146.0 (95\% Cl, 145.1-146.9) per 1000 to $176.3(95 \% \mathrm{Cl}, 175.4-177.2 ;+21 \%)$ and sinus CT from 12.6 (95\% Cl, 12.4-12.8) per 1000 to 15.4 (95\% Cl, 15.2-15.6; +22\%) increased until 2009 and remained stable through 2012.

CONCLUSIONS: Previously increasing same-day sinus and brain CT in Medicare beneficiaries plateaued in 2009, coinciding with the implementation of targeted measures by the Centers for Medicare and Medicaid Services. Same-day imaging continues to increase in the emergency department setting.

ABBREVIATIONS: CMS = Centers for Medicare and Medicaid Services; ED = emergency department; ICD-9 = International Classification of Diseases, Ninth Revision; OP-14 = Outpatient Measure 14 (simultaneous use of brain CT and sinus CT) of the Hospital Outpatient Quality Reporting Program; RIF = Research Identifiable Files

$\mathbf{R}^{\mathrm{i}}$ ising health care costs in the United States have resulted in a number of policy initiatives and professional society campaigns $^{1}$ to use limited resources more judiciously. The rapid increase in spending on medical imaging during the early part of the past decade, ${ }^{2,3}$ along with an increased societal awareness of radiation dose, ${ }^{4}$ has focused those initiatives and campaigns on imaging services. The injudicious use of sinus CT imaging, for example, received considerable attention as part of the multispecialty "Choosing Wisely" initiative. ${ }^{1}$ In response to Congressional leg-

Received June 26, 2015; accepted after revision November 24.

From the Department of Radiology (H.K., M.W.), Neuroradiology Section, Stanford University, Stanford, California; Harvey L. Neiman Health Policy Institute (R.D., J.H., D.H.), Reston, Virginia; Department of Radiology and Imaging Sciences (R.D.), Emory University School of Medicine, Atlanta, Georgia; and Department of Health Administration and Policy (D.H.), George Mason University, Fairfax, Virginia.

Please address correspondence to Max Wintermark, MD, MAS, MBA, Stanford University, Department of Radiology, Neuroradiology Division, 300 Pasteur Dr, Room S047, Stanford, CA 94305-5105; e-mail: Max.Wintermark@gmail.com

http://dx.doi.org/10.3174/ajnr.A4670 islation in 2006, ${ }^{5}$ the Centers for Medicare and Medicaid Services (CMS) also focused on potentially inappropriate sinus CT imaging as part of its outpatient imaging efficiency program. With the rationale that except in specific and narrow circumstances, sameday sinus and brain CT imaging is rarely indicated, CMS established metric Outpatient Measure 14 (OP-14) to track the use by facilities of such same-day imaging. ${ }^{6}$ The goals of the initiative were to improve transparency through public reporting and potentially serve as the basis for value-based payments. Although the overall program was announced in 2006, testing did not begin until 2010 and was not officially implemented until 2012. Nonetheless, even before that official implementation, it received widespread media coverage. ${ }^{7}$

Using just 2 years of CMS Web site data, Rosenkrantz and Doshi ${ }^{8}$ recently reported a relatively low incidence of same-day sinus and brain CT imaging but did not examine how that frequency changed during program rollout or expand their analysis beyond the specific target of the CMS initiative (ie, hospital out- 
patient setting). Thus, despite the attention this program has received in the media, little is known about how physician-testing behavior has changed in association with its implementation.

The aim of our investigation was to study patient-level claims data in the Medicare population, focusing on where same-day sinus and brain CT imaging is performed and how the frequency of same-day studies changed with time before and during the OP-14 measure program implementation.

\section{MATERIALS AND METHODS \\ Data Source}

Our methodology was modeled on that previously described for other imaging procedures. ${ }^{9}$ Under a data-use agreement from the Centers for Medicare and Medicaid Services, we acquired 5\% carrier claims Research Identifiable Files (RIF) from 2004 through 2012, which contain all Medicare Part B fee-for-service claims for a $5 \%$ random sample of Medicare beneficiaries. These files contain detailed information, including date and place of service, procedures performed as identified by Current Procedural Terminology or the Healthcare Common Procedure Coding System code, and beneficiary diagnoses as identified by the International Classification of Diseases, Ninth Revision (ICD-9) code associated with each fee-for-service claim for the approximately 2.5 million beneficiaries present in each year of the data. We received an exemption from the institutional review board of the American College of Radiology for the use of the retrospective administrative data in this study.

\section{Data Parameters}

Sites of service are identified within the 5\% RIF with provider self-reported site-of-service codes. For the purposes of this study, those with site-of-service codes for the physician's office, inpatient hospital, outpatient hospital, and emergency department (ED) settings were specifically identified; all considerably less common sites were grouped together in an "other" site category. All services reported with Current Procedural Terminology codes for sinus CT $(70486,70487,70488)$ and for brain CT (70450, 70460, 70470) were identified annually from 2004 to 2012.

\section{Analysis}

Usage rates for sinus and brain CT in the 5\% random Medicare fee-for-service patient sample were calculated for years 2004 until 2012. All ICD-9 codes associated with each examination were recorded. In concordance with CMS OP-14 methodology, studies performed for the indications of trauma (ICD-9 codes 800-839, 850-854, 860-869, 905-909, 926.11, 926.12, 929, 952, 958-959), infection (ICD-9 Clinical Modification code: 376.01), or known or suspected mass (ICD-9 codes: 140-239) were analyzed separately. These are hereafter termed "exclusion measures," because CMS deemed these diagnoses as ones in which same-day sinus and brain CT examinations may be more appropriate. In addition, the rate of same-day sinus and brain CT was calculated from 2004 until 2012 by using coincident date of service claims, again with and without applied exclusion measures. We classified a sinus and brain CT as "same-day services" if these services were recorded as occurring on the same date of service regardless of whether these were submitted on the same or separate claim

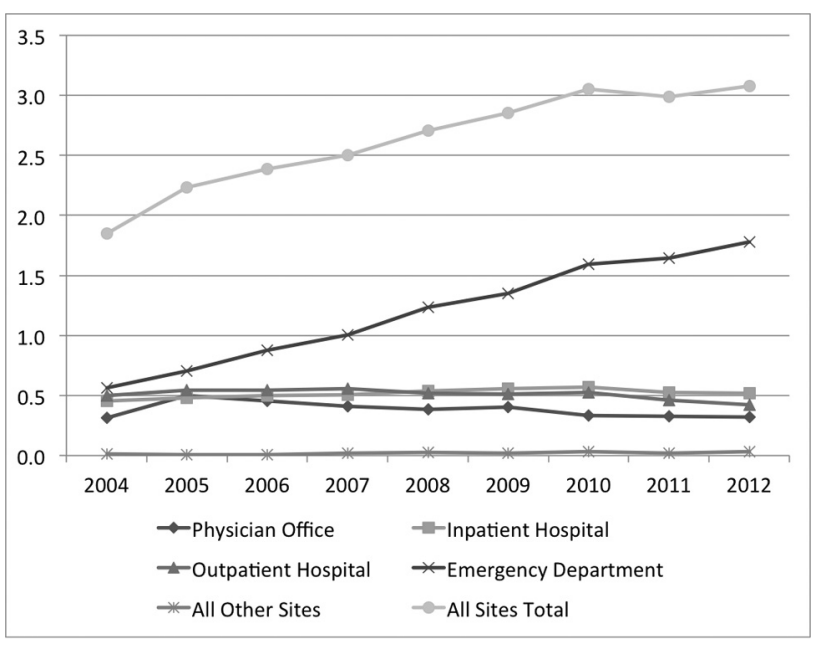

FIG 1. Overall same-day sinus and brain CTs by site of service per 1000 Medicare beneficiaries with exclusion measures applied (exclusion measures are studies performed for trauma, infection, or tumor that were not included in this analysis).

forms. Because physicians and facilities usually submit separate claims for their services, we excluded technical-only claims and included only professional-only and global claims. ${ }^{10}$

Use per 1000 beneficiaries was calculated by dividing the total number of services by the total number of beneficiaries in the $5 \%$ RIF claims files for each year. Data analysis was performed with SAS 9.3 (SAS Institute, Cary, North Carolina).

\section{RESULTS}

From 2004 to 2012, same-day sinus and brain CT examinations per 1000 Medicare beneficiaries were infrequent but increased moderately $(+67 \%)$ with time from 1.85 (95\% CI, 1.78-1.91) in 2004 to 3.08 (95\% CI, 3.00-3.15) in 2012 (Fig 1). A peak in sameday examinations was reached in 2010, 3.05 (95\% CI, 2.98-3.13) studies per 1000 beneficiaries, followed by a relative plateau through 2012, 2.99 (95\% CI, 2.91-3.07) in 2011 and 3.08 (95\% CI, $3.00-3.15)$ in 2012.

With regard to sites of service, small increases in same-day examinations were identified in the physician's office and inpatient hospital settings, from 0.31 (95\% CI, 0.29-0.34) to 0.32 $(95 \% \mathrm{CI}, 0.3-0.35)(+2.4 \%)$ and from 0.45 (95\% CI, 0.42-0.49) to $0.52(95 \% \mathrm{CI}, 0.49-0.55)(+14.3 \%)$, respectively. At the same time, the incidence of same-day examinations in the outpatient hospital setting declined moderately, from 0.50 (95\% CI, 0.47$0.54)$ to $0.42(95 \% \mathrm{CI}, 0.39-0.45)(-16.1 \%)$. The biggest contributor to the overall increase in same-day studies was observed in the emergency department, with a cumulative increase of $215.7 \%$ from 2004 to 2012 , from 0.56 ( $95 \%$ CI, $0.53-0.60$ ) to 1.78 ( $95 \%$ CI, $1.72-1.84)$. In $2004,31 \%$ of all same-day studies were performed in the ED; in 2012, the ED accounted for 58\% of all sameday studies.

With respect to non-same-day services, there was an increase in the overall use of brain CT in the Medicare fee-for-service population from 2004 until 2012, from 146.0 (95\% CI, 145.1-146.9) per 1000 beneficiaries in 2004 to 176.3 (95\% CI, 175.4-177.2) in $2012(+21 \%)$. However, a plateau was achieved in 2009, after which the number of studies stabilized (Fig $2 A$ ). A large percent- 


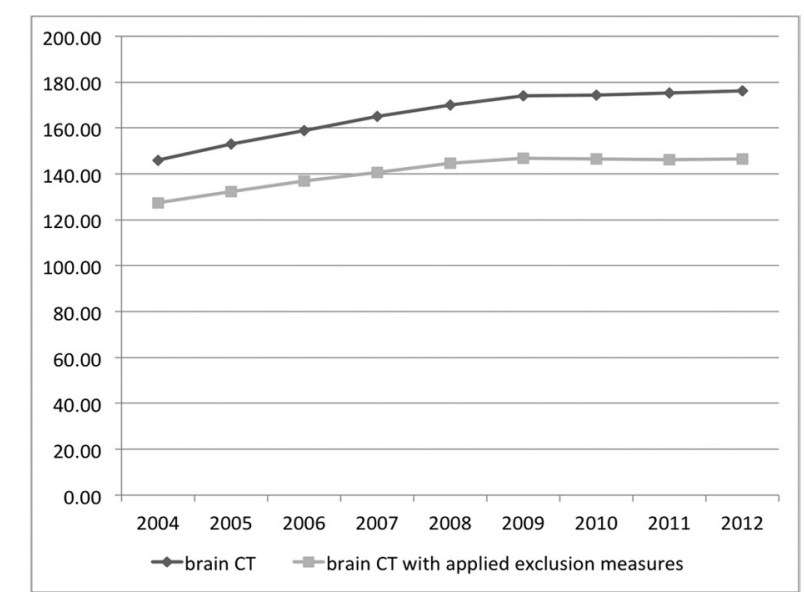

A

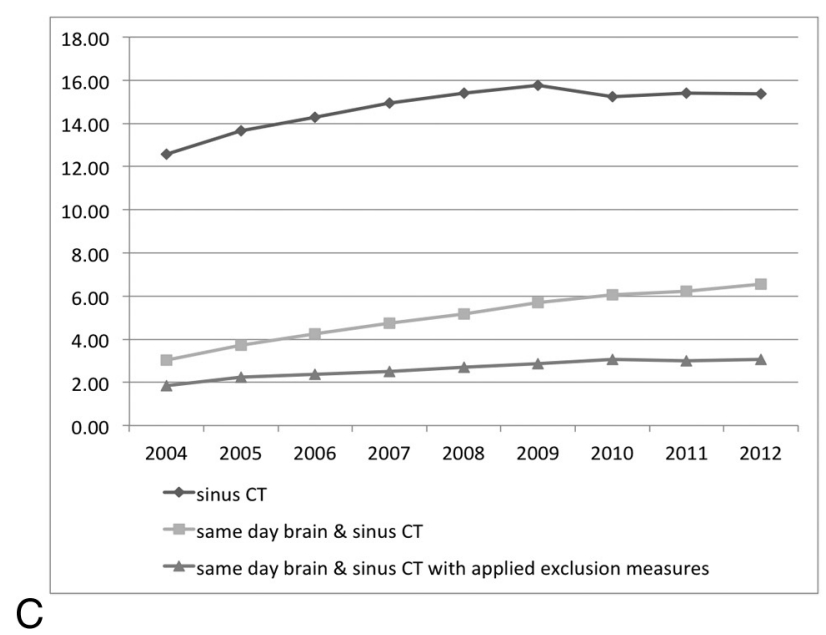

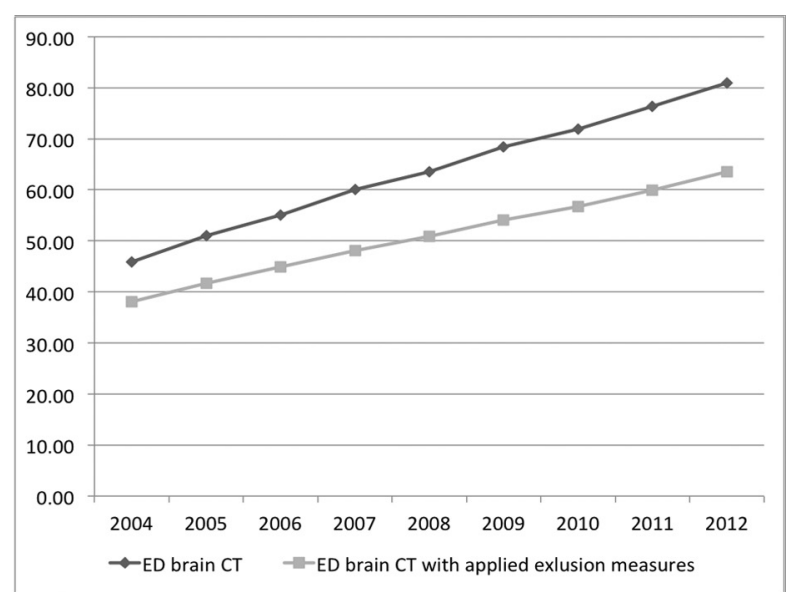

B

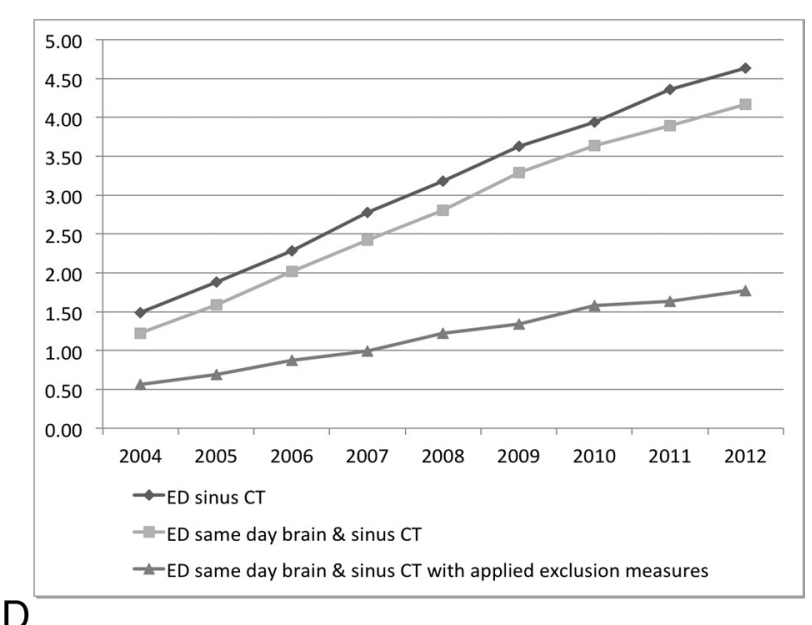

FIG 2. A, All brain CTs and all brain CTs (dark gray line) with applied exclusion measures (light gray line) per 1000 beneficiaries (A). Brain CTs (dark gray line) and brain CTs with applied exclusion criteria (light gray line) performed in the ED (B). Sinus CT (dark gray with rhombs line) and all same-day brain and sinus $C T$, without and with exclusion measures applied, totals are shown in $C$, and those performed in the ED in D. Exclusion measures are CTs that were performed for indications such as trauma, infection, or neoplasm.

age of those studies were performed for a diagnosis other than trauma, infection, or tumor (Fig $2 A, 87 \%$ in 2004 versus $83 \%$ in 2012, $-5 \%)$. Brain CTs performed in the ED showed a steady increase from 2004 to 2012, from 45.8 (95\% CI, 45.5-46.2) per 1000 beneficiaries in 2004 to 80.9 (95\% CI, 80.4-81.4) in 2012 $(+76.5 \%)$. Similar to these findings, though slightly less pronounced, was the number of studies performed for diagnoses other than trauma, infection, or tumor, from 38.1 (95\% CI, 37.7$38.4)$ per 1000 beneficiaries in 2004 to 63.6 (95\% CI, 63.2-64.0) in $2012(+67 \%)$, as illustrated in Fig $2 B$.

The growing use of sinus CT overall slowed in 2009, from 12.6 (95\% CI, 12.4-12.8) per 1000 beneficiaries in 2004 to 15.4 (95\% CI, 15.2-15.6) in $2012(+22 \%$ ); then, it plateaued until 2012 (Fig $2 C)$. However, the frequency of sinus CT performed in the ED continued to increase, from 1.5 (95\% CI, 1.4-1.6) per 1000 beneficiaries in 2004 to 4.6 (95\% CI, 4.6-4.8) in 2012 (+211\%; Fig $2 D)$. Overall, only a minority of all sinus CTs were performed on the same day as brain CT. In the ED setting, in contrast, most sinus CTs were performed with a same-day brain CT (Fig 2C, -D). Although a number of these studies were performed for trauma, infection, or tumor ("exclusion measures" as designated by CMS), a substantial portion of same-day brain CTs and sinus CTs were performed for other indications (gray line with triangles in Fig 2D) from 0.6 (95\% CI, 0.5-0.6) per 1000 beneficiaries in 2004 to $1.8(95 \% \mathrm{CI}, 1.7-1.8)$ in $2012(+216 \%)$.

\section{DISCUSSION}

Although the frequency of same-day sinus and brain CT imaging in the Medicare fee-for-service population is low, it did, in fact, increase in the years leading up to the early stages of implementation of a specific Medicare metric program designed to identify and curb such same-day examinations, supporting the rationale for such an initiative. In the 2 years from metric testing until actual implementation, the frequency of same-day studies plateaued. While we are unable to attribute causation to the OP-14 initiative, our results, nonetheless, suggest that gains are being made in achieving desired testing behavior in accordance with the goals of Medicare. This trend parallels the national stabilization in the use of sinus and brain CT examinations overall (ie, not necessarily on the same day), which began in 2009, suggesting a greater overall awareness of appropriateness in general, which may, in part, be related to concerns about rising costs.

Related to these trends is a new societal emphasis on patient radiation exposure. The effort of professional organizations to 
reduce radiation doses, such as the Image Wisely campaign, ${ }^{11}$ as well as public awareness and media attention ${ }^{12}$ to the potentially harmful side effects of ionizing radiation, may also be impacting effort to reduce use and, therefore, overall population dose. While our study cannot directly link increased radiation awareness to the slowing of growth in medical imaging, it likely contributed to at least part of the decline in imaging studies.

When identifying potential reasons for the increase of sameday studies up to the rollout of the OP-14 policy initiative of Medicare, the most remarkable contributions to overall growth were those performed in the ED setting. Of note, OP-14 applies specifically to the hospital outpatient setting, but the changes in that setting parallel those in all other settings except for the ED. The reasons for this trend and the ED divergence are multifactorial and can only be partially explained by our work. Despite the stabilization in the overall use of sinus and brain CT imaging since 2009, volume in the ED for CT overall continues to rise. ${ }^{13}$ Our observations for sinus and brain imaging parallel this overall discordant trend, which may, in part, explain the continued increased same-day sinus and brain CT imaging in the ED setting. The national trends reported by Levin et $\mathrm{al}^{13}$ used aggregated (ie, no patient-specific information) claims data. Our use of more detailed claims-level data permitted us to examine reported indications for individual examinations, providing additional perspectives. Many simultaneous ED examinations, for example, were ordered in the setting of trauma, infection, or tumor. In such situations, same-day examinations are more appropriate because they include clinical scenarios other than generic headache, such as suspected or known masses involving the paranasal sinuses, face, nasopharynx, or skull base and suspected intracranial extension of infectious processes involving the sinuses.

Nonetheless, almost half of same-day sinus and brain CT scans were not ordered for these 3 specific recognized groups of indications. While our study was not designed to investigate other indications, we can propose several potential explanations.

In our study, we applied exclusion measures for patients with trauma, infection, or tumor on the basis of the ICD codes detailed in the OP-14 criteria, to exclude studies from our analysis that clearly warrant both a sinus and a head CT. Using exclusion criteria based on ICD codes, however, has limitations. While primary ICD codes incorporate the main diagnoses, many additional diagnoses may exist but not be captured through the ICD coding system because coders may focus on only key diagnoses necessary to establish medical necessity in the eyes of a payer. For example, a patient might have a syncopal event with a fall and present to the ED to evaluate presenting symptoms of dizziness. The ED provider may have ordered a sinus CT and brain CT as part of a minor trauma work-up. The traumatic event, however, may not have been captured by the ICD coding, which only included the syncope and/or altered mental status. An additional limitation is that by using administrative claims data, actual sites of service cannot be verified; we thus relied on self-reported sites of service. Because some patients in the ED are subsequently hospitalized, some ED claims could be erroneously reported by using the inpatient hospital setting as the site of service, thus underestimating ED volumes.

Another indication that may account for same-day sinus and brain CT imaging outside the context of trauma, infection, and tumor is headache. Wang and You ${ }^{14}$ found that approximately one-quarter of patients without trauma who underwent brain CT in the ED presented with headaches. Because sinusitis is a frequent source of headaches, many same-day studies may have been performed to investigate both the sinuses and brain as causes of headaches, resulting in unnecessary duplicate examinations. Educating ordering physicians that portions of the paranasal sinuses could be covered on routine brain CT examinations or adjusting sinonasal coverage on brain CTs when sinusitis is of clinical concern could help decrease the frequency of duplicate examinations in the work-up of headache. On the other hand, increasing effort to limit the radiation dose to the lens on routine head CT examinations by patient positioning and gantry tilt leads to decreased paranasal sinus coverage, which further underpins the need to tailor the imaging protocols to the clinical and anatomic ROIs.

Independent of the clinical scenarios leading to the ordering of same-day sinus and brain CT examinations, our data clearly demonstrate that the ED is the setting most responsible for the recent increase in simultaneous sinus and brain CT imaging. Multiple studies have already demonstrated an increased use of brain CT in the ED for minor head trauma. ${ }^{15-18}$ Emergency physicians are exposed to growing pressure to address increasing patient loads despite ongoing demands for faster turnaround times and quicker ED and hospital discharges. This likely leads ED physicians to rely more heavily on imaging to achieve quicker diagnoses. While there is no easy solution for this problem, better communication between emergency physicians and radiologists with closer interaction through increasing physical proximity may help both specialties in collaborating to ensure the most appropriate use of imaging for the work-up of patients in the ED. ${ }^{19}$ Growing as well are clinical decision-support tools, which may help emergency physicians more effectively leverage finite imaging resources. ${ }^{20,21}$

We acknowledge certain limitations to our study. First, the datasets included in our study are from 2004 to 2012 and do not include the recent years 2013 and 2014, which were not available to the authors for analysis at the time of publication. Omitting these data points clearly limits the ability of this study to report on the long-term effects of the OP-14 measure on the performance of same-day brain and sinus CTs. Second, the descriptive methodology of this publication limits the ability to attribute causation to the changes in same-day brain and sinus imaging. Further studies that include more longitudinal data points and incorporate regression-based models may be necessary to prove such causation.

\section{CONCLUSIONS}

Same-day brain and sinus CT scans in the Medicare population increased until the year 2009 when a plateau was reached, which coincided with the implementation of targeted measures by CMS to reduce imaging over-utilization. While no causal relationship between the CMS measure and the plateauing of same-day studies could be established, emergency departments were identified as the biggest driver of same-day examinations. Targeted strategies to reduce same-day orders out of the emergency room may have a significant impact for future appropriate imaging utilization.

AJNR Am J Neuroradiol 37:1000-04 Jun 2016 www.ajnr.org 1003 
Disclosures: Max Wintermark—UNRELATED: Board Membership: GE National Football League advisory board.

\section{REFERENCES}

1. Cassel CK, Guest JA. Choosing wisely: helping physicians and patients make smart decisions about their care. JAMA 2012;307: 1801-02 CrossRef Medline

2. Iglehart JK. The new era of medical imaging-progress and pitfalls. N Engl J Med 2006;354:2822-28 CrossRef Medline

3. Iglehart JK. Health insurers and medical-imaging policy: a work in progress. N Engl J Med 2009;360:1030-37 CrossRef Medline

4. Brenner DJ, Hall EJ. Computed tomography: an increasing source of radiation exposure. $N$ Engl J Med 2007;357:2277-84 CrossRef Medline

5. Tax Relief and Health Care Act of 2006, Pub L No. 109-432 (2006)

6. Medicare.gov Hospital Compare. http://www.medicare.gov/hospital compare/search.html. Accessed January 5, 2015

7. Bogdanich W, McGinty JC. Medicare claims show overuse of CT scanning. New York Times. June 17, 2011. http://www.nytimes.com/ 2011/06/18/health/18radiation.html. Accessed January 5, 2015

8. Rosenkrantz AB, Doshi A. Characterizing the performance of the nation's hospitals in the Hospital Outpatient Quality Reporting Program's imaging efficiency measures. J Am Coll Radiol 2015;12: 166-73 CrossRef Medline

9. Duszak R Jr, Allen B Jr, Hughes DR, et al. Emergency department CT of the abdomen and pelvis: preferential utilization in higher complexity patient encounters. J Am Coll Radiol 2012;9:409-13 CrossRef Medline

10. Levin DC, Rao VM, Parker L. Physician orders contribute to hightech imaging slowdown. Health Aff (Millwood) 2010;29:189-95 CrossRef Medline

11. Brink JA, Amis ES Jr. Image wisely: a campaign to increase awareness about adult radiation protection. Radiology 2010;257:601-02 CrossRef Medline
12. Bogdanich W. Radiation boom: newspaper series. 2010-2015. New York Times. March 17, 2015. http://topics.nytimes.com/top/news/us/ series/radiation_boom/index.html. Accessed January 5, 2015

13. Levin DC, Rao VM, Parker L, et al. Continued growth in emergency department imaging is bucking the overall trends. J Am Coll Radiol 2014;11:1044-47 CrossRef Medline

14. Wang X, You JJ. Head CT for nontrauma patients in the emergency department: clinical predictors of abnormal findings. Radiology 2013;266:783-90 CrossRef Medline

15. Haydel MJ, Preston CA, Mills TJ, et al. Indications for computed tomography in patients with minor head injury. N Engl J Med 2000; 343:100-05 CrossRef Medline

16. Jagoda AS, Bazarian JJ, Bruns JJ Jr, et al; American College of Emergency Physicians, Centers for Disease Control and Prevention. Clinical policy: neuroimaging and decision making in adult mild traumatic brain injury in the acute setting. Ann Emerg Med 2008;52: 714-48 CrossRef Medline

17. Smits M, Dippel DW, de Haan GG, et al. External validation of the Canadian CT Head Rule and the New Orleans Criteria for CT scanning in patients with minor head injury. JAMA 2005;294:1519-25 CrossRef Medline

18. Stiell IG, Clement CM, Rowe BH, et al. Comparison of the Canadian CT Head Rule and the New Orleans Criteria in patients with minor head injury. JAMA 2005;294:1511-18 CrossRef Medline

19. Tillack AA, Borgstede JP. An evaluation of the impact of clinically embedded reading rooms on radiologist-referring clinician communication. J Am Coll Radiol 2013;10:368-72 CrossRef Medline

20. Blackmore CC, Mecklenburg RS, Kaplan GS. Effectiveness of clinical decision support in controlling inappropriate imaging. J Am Coll Radiol 2011;8:19-25 CrossRef Medline

21. Zafar HM, Mills AM, Khorasani R, et al. Clinical decision support for imaging in the era of the Patient Protection and Affordable Care Act. J Am Coll Radiol 2012;9:907-18.e5 CrossRef Medline 